\title{
Hierarchical Scaled-States Direct Predictive Control of Synchronous Reluctance Motor Drives
}

\author{
Riccardo Antonello, Member, IEEE, Matteo Carraro, Luca Peretti and Mauro Zigliotto, Member, IEEE
}

\begin{abstract}
This paper presents the design and experimental validation of a finite-state direct predictive control for synchronous reluctance motor drives. The main features are the hierarchical selection policy of the optimal voltage vector and the dynamic scaling of the voltage amplitude which keeps the current ripple limited even in presence of low switching frequencies, as required by medium and high-power applications. The implementation is simple, intuitive and low-demanding. The study is fully supported by experimental evidences.
\end{abstract}

Index Terms-Direct Predictive Control, Synchronous Reluctance Motors.

\section{INTRODUCTION}

$\mathbf{O}$ VER the past years, Model Predictive Control (MPC) techniques gained increasing popularity in the research field of advanced control techniques for power electronics converters and electric drives [1]-[4]. Their distinctive features include the overcoming of the conventional cascaded control structures, the possibility of incorporating an optimality criterion in the control strategy [5] and the capability of dealing with system constraints in a easy and straightforward manner [6], [7]. The main drawback of MPC still remains the heavy computational requirements, because a constrained optimisation problem has to be solved at every sampling time. Some solutions are available to limit the computational effort required by MPC. In the Explicit MPC technique, under certain limiting hypotheses on the system model (linear, time invariant model), constraints (linear inequalities) and cost function (quadratic cost function), an explicit form for the control law is derived, so that it can be precomputed offline [8], [9]. However, the exclusion of nonlinear systems poses serious limitations to the explicit MPC applicability.

As a valid alternative, Direct Predictive Control (DPC) uses a finite set of control inputs [10]-[13], so that the optimisation problem can be solved online by iteratively testing the cost function and constraints against all the available inputs, and then opting for the admissible value yielding the minimum cost. This approach is well suited for inverter-fed electric drives applications, given the limited set of state voltage vectors that can be generated with a three-phase voltage source inverter.

Manuscript received June 8, 2015; revised November 13, 2015 and January 7, 2016; accepted February 4, 2016.

R. Antonello and M. Zigliotto are with the Dept. of Management and Engineering, University of Padova, Italy. (e-mail: \{riccardo.antonello, mauro.zigliotto\}@unipd.it).

M. Carraro is with Sael Srl, 36040 Torri di Quartesolo (VI), Italy. (e-mail: carraro@sael.it)

L. Peretti is with the Dept. of Electrical Systems, ABB Corporate Research, 72178 Västerås, Sweden (e-mail: luca.peretti@se.abb.com).
The use of state vectors only, without pulse width modulation, produces a sensitive increment of the phase current ripple [14]. Except for low DC bus voltage applications [15], the ripple can be reduced by increasing the switching frequency time, but an upper limit derives by both the computational time required to solve the optimisation problem and by the characteristics of the power switches. This limitation can be partly relaxed by adopting faster computational devices, such as parallel processors or FPGA units [16]. However, regardless of any computational aspect, there are other situations, such as in medium and high power applications, where the inverter switching frequency must be kept small, to comply with efficiency and thermal constraints. The alternative approach for ripple mitigation, i.e. the growth of inverter voltage levels, results in the exponential increase of the number of inverter states to be tested at each sampling time, which rapidly makes the prediction unfeasible.

In this paper, a novel design of a DPC oriented to medium and high power Synchronous Reluctance Motor Drives (SynRM) is presented. In the proposed solution, named Hierarchical Direct Predictive Control (HDPC), the online iterative search for the optimal solution of a conventional DPC implementation is replaced by a multi-level decisional structure based on a hierarchical set of tests, whose aim is to identify the optimal solution through successive refinements of the set of admissible inputs. This alternative implementation adds flexibility to the DPC scheme, enabling to set priorities among the constraints to satisfy when searching for the optimal solution. To keep a reasonable current ripple, while maintaining a low algorithm complexity, the proposed HDPC design is then further improved by introducing the use of "scaled-states" voltage vectors. As the name suggests, it consists in scaling the amplitude of the inverter state voltage vectors by means of an attenuation factor that is adjusted according to the drive operation. The scaling is computed before each prediction cycle, so that it does not increase the number of states to be tested, opposite to the multi-level inverter approach [17].

The paper is organised as follows. The mathematical model of the SynRM used for state prediction by the the proposed HDPC scheme is presented in Sec. II. Both the cross-coupling and saturation effects in the magnetic flux linkages are accounted for in the model, to improve its prediction accuracy. The details about the HDPC structure and implementation are reported in Sec. III. These include the descriptions of the hierarchical decisional structure exploited for the selection of the optimal input, and the voltage scaling policy adopted to limit the current ripple without any further increase in the number of input alternatives. The effectiveness of the proposed 
HDPC was tested both by simulations and experiments on a $11 \mathrm{~kW}$ SynRM prototype. The results are reported in Sec. IV and Sec. V. Some final consideration and remarks are then pointed out in Sec. VI.

\section{Synchronous Reluctance Motor Model}

\section{A. Continuous-time model}

Any predictive controller relies on a mathematical model of the system to be controlled to plan the future control action. Modelling accuracy is essential for avoiding wrong predictions that would definitely yield a degradation of the control performances. Yet, a trade-off with model complexity is required to make the state prediction feasible in real time.

In order to comply with both requirements, the drive model chosen for the proposed HDPC is that of a Synchronous Reluctance Motor (SynRM) expressed in the synchronous $d q$ frame, with the $d$ axis aligned along the direction of minimum reluctance.

$$
\frac{d \boldsymbol{\lambda}}{d t}=\boldsymbol{u}-R \boldsymbol{i}-j \omega_{m e} \boldsymbol{\lambda}
$$

where $\boldsymbol{\lambda}=\lambda_{d}+j \lambda_{q}, \boldsymbol{i}=i_{d}+j i_{q}$ and $\boldsymbol{u}=u_{d}+j u_{q}$ are, respectively, the flux linkage, current and voltage space vectors, and $\omega_{m e}=p \omega_{m}$ is the electromechanical rotor speed, with $p$ denoting the number of motor pole pairs, and $\omega_{m}$ the mechanical rotor speed. The two flux components $\lambda_{d}$ and $\lambda_{q}$ are modelled as two nonlinear functions depending on both current components $i_{d}$ and $i_{q}$, i.e.

$$
\lambda_{d}=f_{d}\left(i_{d}, i_{q}\right), \quad \lambda_{q}=f_{q}\left(i_{d}, i_{q}\right)
$$

to account for both cross-coupling and saturation effects. The two functions are graphically depicted in Fig. 1.

The mechanical dynamics is modelled as that of a simple lumped inertia

$$
\frac{d \omega_{m}}{d t}=-\frac{B_{m}}{J_{m}} \omega_{m}+\frac{1}{J_{m}}\left(\tau_{m}-\tau_{L}\right)
$$

where $J_{m}$ is the rotor inertia, $B_{m}$ the viscous friction coefficient, $\tau_{m}$ the reluctance torque produced by the motor and $\tau_{L}$ a generic load torque. The reluctance torque is equal to

$$
\tau_{m}=(3 / 2) p \Im(i \bar{\lambda})
$$

where $(\cdot)$ and $\Im(\cdot)$ denote the complex conjugate and imaginary part of the argument. Ultimately, the SynRM dynamical model (1)-(4) can be schematically represented with the block diagram shown in Fig. 2.
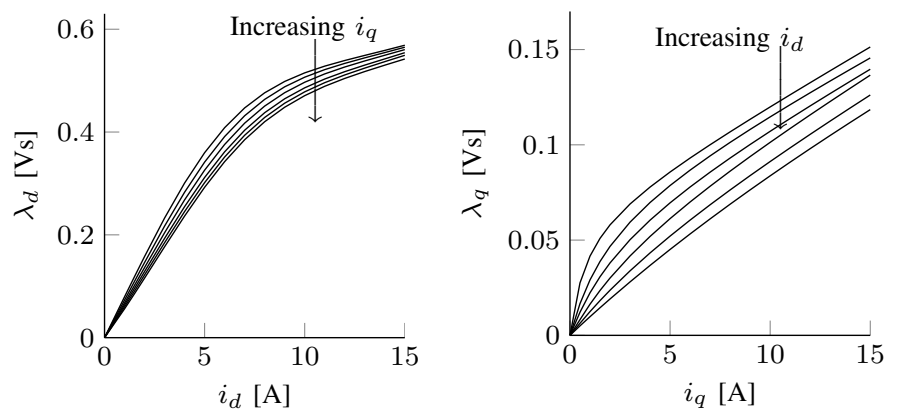

Fig. 1. Measured magnetic flux linkages vs currents in $d q$ frame.

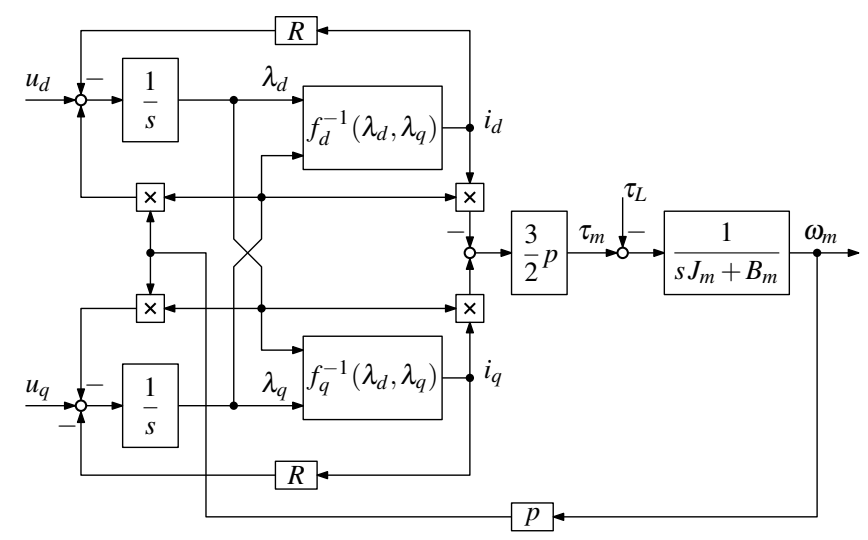

Fig. 2. Block diagram of the SynRM.

\section{B. Model discretization}

For the implementation of the state predictor, the continuous-time model introduced above needs to be discretized. In the following, let $x_{k}$ denotes the sampled version of a generic variable $x(t)$ at the sampling instant $t=k T_{s}$, with $T_{s}$ representing the sampling period. By introducing the average operator

$$
\langle x\rangle_{k} \triangleq \frac{1}{T_{s}} \int_{k T_{s}}^{(k+1) T_{s}} x(t) d t
$$

the integration of both sides of (1) over the time interval $\left[k T_{s},(k+1) T_{s}\right]$ yields

$$
\boldsymbol{\lambda}_{k+1}=\boldsymbol{\lambda}_{k}+\langle\boldsymbol{u}\rangle_{k}-R T_{s}\langle\boldsymbol{i}\rangle_{k}-j T_{s}\left\langle\omega_{m e} \boldsymbol{\lambda}\right\rangle_{k}
$$

By assuming that

$$
\langle i\rangle_{k} \approx \frac{\boldsymbol{i}_{k+1}+\boldsymbol{i}_{k}}{2}, \quad\left\langle\omega_{m e} \boldsymbol{\lambda}\right\rangle_{k} \approx \omega_{m e, k} \frac{\boldsymbol{\lambda}_{k+1}+\boldsymbol{\lambda}_{k}}{2}
$$

and by imposing a constant voltage vector $\boldsymbol{u}$ on the whole sampling period, then (6) can be approximated as follows

$$
\begin{array}{r}
\boldsymbol{\lambda}_{k+1} \approx \boldsymbol{\lambda}_{k}+\boldsymbol{u}_{k}-R T_{s}\left(\boldsymbol{i}_{k+1}+\boldsymbol{i}_{k}\right) / 2-\cdots \\
\cdots-j \omega_{m e, k} T_{s}\left(\boldsymbol{\lambda}_{k+1}+\boldsymbol{\lambda}_{k}\right) / 2
\end{array}
$$

Since the current vector $\boldsymbol{i}_{k+1}$ has a nonlinear dependence on the flux linkage $\boldsymbol{\lambda}_{k}$, the only way to solve (8) for $\boldsymbol{\lambda}_{k+1}$ is to resort to numerical methods. This approach is unsuitable for the implementation of a fast predictor for real time applications. However, accepting that the average current $\langle\boldsymbol{i}\rangle_{k}$ in (6) can be approximated by its instantaneous value $\boldsymbol{i}_{k}$, then the following closed form solution for $\boldsymbol{\lambda}_{k+1}$ can be obtained

$$
\boldsymbol{\lambda}_{k+1} \approx \frac{1-j \omega_{m e, k} T_{s} / 2}{1+j \omega_{m e, k} T_{s} / 2} \boldsymbol{\lambda}_{k}+\frac{\boldsymbol{u}_{k}-R \boldsymbol{i}_{k}}{1+j \omega_{m e, k} T_{s} / 2}
$$

For the discretization of the mechanical dynamics, the average operator (5) is applied to both sides of (3), yielding

$$
\omega_{m, k+1}=\omega_{m, k}-\frac{B_{m} T_{s}}{J_{m}}\left\langle\omega_{m}\right\rangle_{k}+\frac{T_{s}}{J_{m}}\left(\left\langle\tau_{m}\right\rangle_{k}-\left\langle\tau_{L}\right\rangle_{k}\right)
$$

By using the following approximation

$$
\left\langle\omega_{m}\right\rangle_{k} \approx \frac{\omega_{m, k}+\omega_{m, k+1}}{2}
$$


within (10), and then solving for $\omega_{m, k+1}$, it holds that

$$
\omega_{m, k+1} \approx\left(1-\frac{B_{m} T_{s}}{J_{m}}\right) \omega_{m, k}+\frac{T_{s}}{J_{m}}\left(\left\langle\tau_{m}\right\rangle_{k}-\left\langle\tau_{L}\right\rangle_{k}\right)
$$

where it has been additionally assumed that $B_{m} J_{m} /\left(2 T_{s}\right) \ll$ 1. Since the load torque is typically a slowly varying quantity compared to the sampling period, its average value in (12) can be reasonably approximated as

$$
\left\langle\tau_{L}\right\rangle_{k} \approx \tau_{L, k}
$$

As for the reluctance torque, its average value is approximated similarly to (11), i.e.

$$
\left\langle\tau_{m}\right\rangle_{k} \approx \frac{\tau_{m, k}+\tau_{m, k+1}}{2}
$$

\section{HDPC ARCHITECTURE}

\section{A. General overview}

The proposed control algorithm belongs to the family of the direct predictive control (DPC) schemes. It implies that at every control step, the algorithm iteratively tests all the available control inputs in order to select the one yielding the optimal response (according to a specified criterion) over the prediction horizon. In this paper, the main difference with respect to standard DPCs is the presence of a multi-level decisional strategy for the selection of the optimal control input, which replaces the conventional minimisation of a certain cost function over the set of admissible solutions. In practice, the optimal solution is attained through successive refinements of the set of admissible inputs, that are operated according to a hierarchical set of tests.

As in conventional DPCs, both the number of available control inputs and the length of the prediction horizon should be limited to avoid an excessive computational load. With a two-level, three-phases voltage source inverter, the minimal choice corresponds to use the state vectors set

$$
\mathcal{U}_{1}=\{0\} \cup\left\{(2 / 3) U_{d c} e^{j n \pi / 3}\right\}_{n=0, \ldots, 5}
$$

with $U_{d c}$ being the DC link voltage. A reduced number of voltage vectors usually yield an excessive current ripple. Two countermeasures are adopted in this paper for its mitigation:

1) the minimal set of available voltage vectors given in (15) is augmented with six additional vectors

$$
\mathcal{U}_{2}=\left\{(2 / 3) U_{d c} e^{j(n \pi / 3+\pi / 6)}\right\}_{n=0, \ldots, 5}
$$

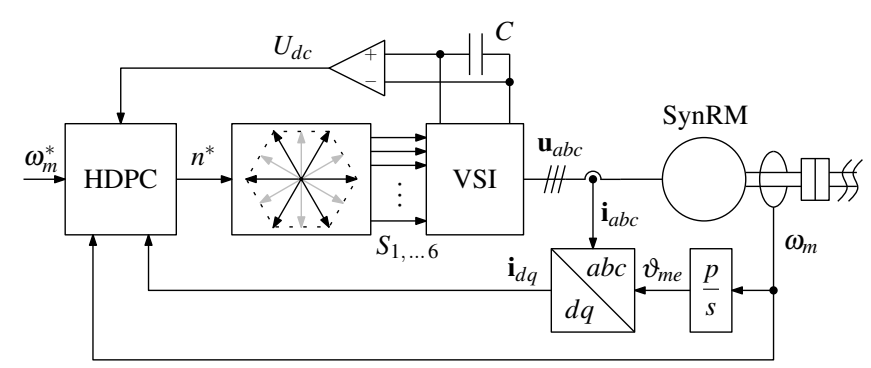

Fig. 3. Block diagram of the overall HDPC scheme. to form the extended set $\mathcal{U}=\mathcal{U}_{1} \cup \mathcal{U}_{2}$ of 13 elements. Every voltage vector in $\mathcal{U}_{2}$ is generated by alternatively applying the two adjacent vectors in $\mathcal{U}_{1}$, each for half of the sampling period [18].

2) the state voltage vectors are scaled by a factor $0<\kappa \leq$ 1 , which is continuously adjusted according to the drive operating condition (scaled-states mechanism).

Regarding the selection of the prediction horizon length, it can be noticed that the voltage vector selected at step $k$ is actually generated by the inverter during the next sampling interval, so that its effects on the motor currents and speed can only be observed respectively at steps $k+1$ and $k+2$. Therefore, the minimum prediction horizon length is $N_{y}=2$, and this is the choice for the HDPC implementation presented in this paper. The block diagram of the overall HDPC-based electric drive is illustrated in Fig. 3. The details of the hierarchical decisional strategy implemented within the HDPC block for the selection of the optimal voltage vector are illustrated in the next sections, together with those of the state predictor upon which such strategy is based.

\section{B. State prediction}

At every control step $k$, the two-step ahead prediction of the system state is obtained by performing the following sequence of computations:

1) State estimation at step $k$ : given the current measurement $\boldsymbol{i}_{k}$, the flux estimation $\boldsymbol{\lambda}_{k}$ is first obtained by evaluating (2), and then used in (4) to retrieve the torque estimate $\hat{\tau}_{m, k}$.

2) State prediction at step $k+1$ : given the current measure $\boldsymbol{i}_{k}$, the flux estimate $\boldsymbol{\lambda}_{k}$, and the voltage reference $\boldsymbol{u}_{k}$ (computed at step $k-1$ ), the flux linkage prediction $\hat{\boldsymbol{\lambda}}_{k+1}$ is obtained by evaluating (9). Then, by using the inverse of the maps (2), it is possible to retrieve the current prediction $\hat{\boldsymbol{i}}_{k+1}$. By replacing the flux and current predictions so computed within (4), the torque prediction $\hat{\tau}_{m, k+1}$ is obtained. Finally, the speed prediction $\hat{\omega}_{m, k+1}$ is obtained by evaluating (12) with the speed measure $\omega_{m, k}$ available at step $k$ and the load torque estimate (13) provided by the disturbance observer described in the next Sec. III-C. The average reluctance torque in (12) is obtained from (14), using the observed torque values at steps $k$ and $k+1$.

3) State prediction at step $k+2$ : the procedure outlined at step $k+1$ is repeated at step $k+2$, using the values predicted at step $k+1$ instead of those observed at time $k$. The prediction is repeated for every voltage vector of the extended set $\mathcal{U}$.

\section{Load torque observer}

The estimation of the load torque is performed with the classical disturbance observer (DOB) scheme reported in [19]. At step $k$, the load torque is estimated as

$$
\hat{\tau}_{L}=\hat{\tau}_{m, k}-\hat{\tau}_{0, k}
$$

where $\hat{\tau}_{m, k}$ is the estimate of the actual torque produced by the motor, and $\hat{\tau}_{0, k}$ is the torque required to drive the unloaded nominal mechanical system (3) at the measured speed. After backward Euler's discretization, $\hat{\tau}_{0, k}$ is given by

$$
\hat{\tau}_{0, k}=J_{m} \frac{\omega_{m, k}-\omega_{m, k-1}}{T_{s}}+B_{m} \omega_{m, k}
$$


The undesired amplification of the speed measurement noise produced by the discrete derivative in (18) is partially rejected by a low-pass filter. A cut-off frequency $f_{c}=50 \mathrm{~Hz}$ was selected as a trade-off between noise rejection and the observer bandwidth. It is worth to note that the HDPC produces a satisfactory response even in presence of a mechanical parameter mismatch. In fact, any discrepancy between the nominal and actual mechanical dynamics produces an extra contribution in the load torque estimate (17), that the HDPC will compensate. In this way, the final effect is that the whole control system operates as if the motor behaves as its nominal model, as well known and documented in literature [19].

\section{Input selection policy}

At every step $k$, the optimal voltage vector to be applied at next sampling interval is determined through a hierarchical sequence of tests, based on the state predicted at step $k+2$. By evaluating the tests sequentially, a successive refinement of the set of admissible voltage vectors is carried out, finally leading to the optimal solution. In defining the sequence of tests, the highest priority is given to the regulation of the $d$-axis current within a specified error threshold, under the mandatory constraint imposed by the motor current limits. This choice corresponds to preserve a certain magnetic flux level in the machine, regardless of its operation, so that a faster torque response can be obtained. Among all the vectors satisfying the current regulation condition, the next selection is operated according to the speed regulation requirement, and then on the reduction of the $q$-axis current ripple. The multilevel decisional process can be detailed as follows:

1) within the set of available voltage vectors $\boldsymbol{u}^{n}$ with $n \in$ $\mathcal{N}_{0}=\{1,2, \ldots, 13\}$, select only those in $\mathcal{N}_{1} \subseteq \mathcal{N}_{0}$ producing a current vector $\hat{\boldsymbol{i}}_{k+2}^{n}$ at step $k+2$ satisfying the current limit

$$
\left|\hat{\boldsymbol{i}}_{k+2}^{n}\right|<I_{N}
$$

where $I_{N}$ is the motor nominal current. If none exists, then select the optimal voltage vector as the one minimising the magnitude of the predicted current vector, i.e. the one whose index is

$$
n^{*}=\underset{n \in \mathcal{N}_{0}}{\arg \min }\left|\hat{\boldsymbol{i}}_{k+2}^{n}\right|
$$

2) among the voltage vectors selected at point 1 , consider those in $\mathcal{N}_{2} \subseteq \mathcal{N}_{1}$ that satisfy the condition

$$
\left|e_{d, k+2}\right|<e_{d, \max }
$$

where $e_{d, k+2}=i_{d, k+2}^{*}-\hat{i}_{d, k+2}$ is the predicted direct current error at step $k+2$. In addition to the vectors satisfying (21), the vectors that comply with the condition

$$
\left|e_{d, k+2}\right|<\left|e_{d, k+1}\right|
$$

are also considered to extend the set of admissible vectors passed to the next decisional level. In fact, operating a drastic restriction in this first step of evaluation could jeopardise the remaining choices. If no vectors satisfying

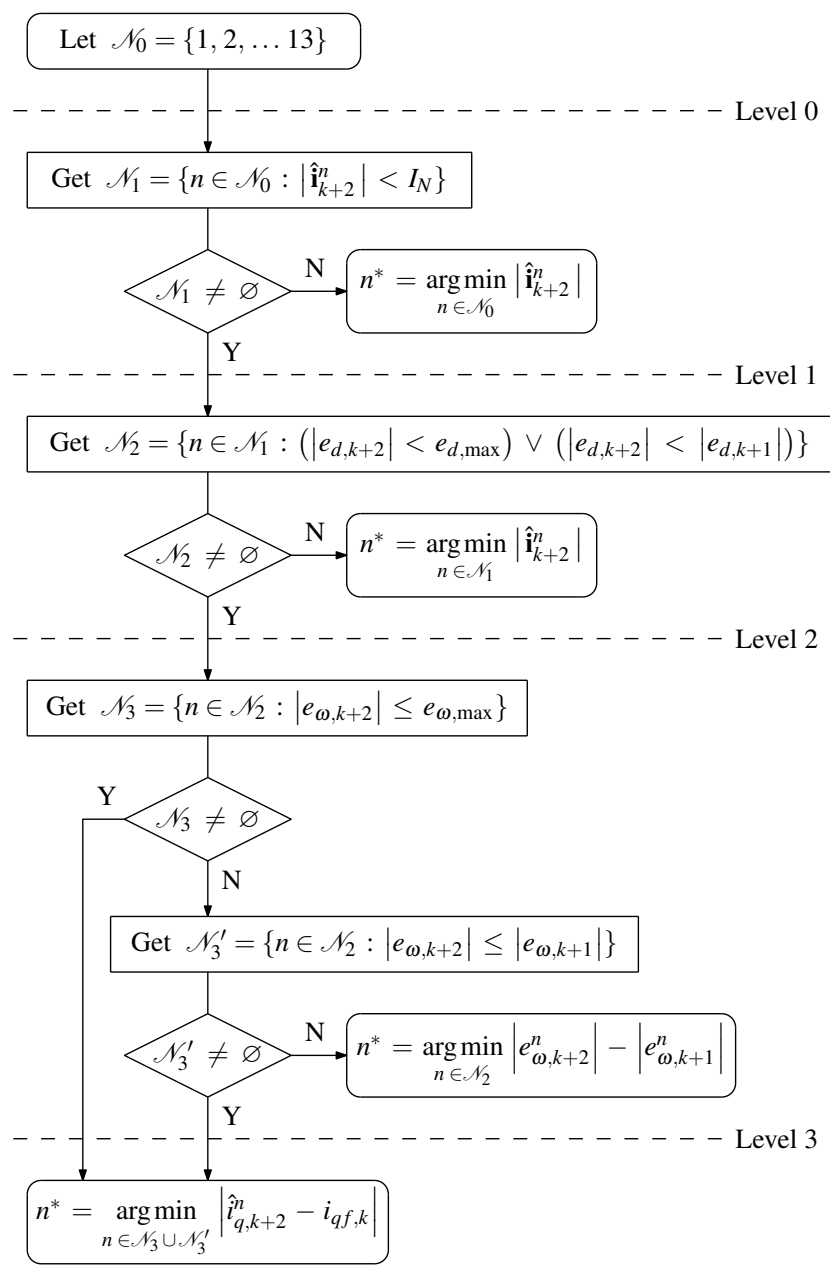

Fig. 4. Hierarchical decisional process for optimal input selection.

(21) or (22) exist, then select the optimal voltage vector as in point 1 (but restricted to the set $\mathcal{N}_{1}$ ).

3 ) among the voltage vectors selected at point 2 , choose those in $\mathcal{N}_{3} \subseteq \mathcal{N}_{2}$ that satisfy the condition

$$
\left|e_{\omega, k+2}\right| \leq e_{\omega, \max }
$$

where $e_{\omega, k+2}=\omega_{k+2}^{*}-\hat{\omega}_{k+2}$ is the predicted speed error at step $k+2$. If none exists, then select those in $\mathcal{N}_{3}^{\prime} \subseteq \mathcal{N}_{2}$ that decrease the speed error, i.e.

$$
\left|e_{\omega, k+2}\right| \leq\left|e_{\omega, k+1}\right|
$$

If neither (23) nor (24) can be satisfied, then the optimal voltage vector is that producing the minimum increase of the speed error, i.e.

$$
n^{*}=\underset{n \in \mathcal{N}_{2}}{\arg \min }\left|e_{\omega, k+2}^{n}\right|-\left|e_{\omega, k+1}^{n}\right|
$$

4) among the voltage vectors selected at point 3 , the optimal is chosen as that minimising the deviation of the predicted $q$-axis current from its "moving average" value, i.e.

$$
n^{*}=\underset{n \in \mathcal{N}_{3} \cup \mathcal{N}_{3}^{\prime}}{\arg \min }\left|\hat{i}_{q, k+2}^{n}-i_{q f, k}\right|
$$


where $i_{q f, k}$ is the output at step $k$ of a 80-taps moving average filter.

A flowchart representation of the hierarchical decisional process described above is reported in Fig. 4.

\section{E. State vectors scaling policy}

In addition to increase the number of available inverter states to reduce the current ripple, further ripple mitigation is obtain by scaling the state voltage vectors amplitude by a factor $\kappa$. In practice, $\kappa$ is computed to get just the sufficient supply voltage for sustaining the motor operation at the current speed and load torque conditions. This is roughly obtained by discretizing (1) and solving for $\boldsymbol{u}$ :

$$
\boldsymbol{u}_{\mathrm{req}, k} \approx R \boldsymbol{i}_{k}+j\left(\lambda_{q, k+1}-\lambda_{q, k}\right) / T_{s}+j \omega_{m e, k} \boldsymbol{\lambda}_{k}
$$

It is worth to note that since $\lambda_{d}$ is regulated to an almost constant value, its derivative is omitted in the computation of (27). At every step $k$, the scaling factor $\kappa$ is updated as follows

$$
\kappa=\left|\boldsymbol{u}_{\text {req, }, k}\right| /\left(2 / 3 U_{d c}\right)
$$

\section{Simulation RESUltS}

Several numerical simulations were carried out to test the HDPC response under different loading and parameter uncertainty conditions. The SynRM model used for the tests includes the nonlinear magnetic flux linkage characteristics shown in Fig. 1, the position and current measurement quantisations, and the voltage saturations. The magnetic model was obtained by extensive experimental commissioning measurement campaigns based on state of the art methods [20]-[22]. The nameplate data of the SynRM used both for simulations and experimental tests are reported in Tab. I in the Appendix. For a closer match of the simulation model to the real experimental setup, a $T_{s}=250 \mu$ s sampling time was selected for the HDPC. The quantisation due to the position encoder (16bit resolution per rotor mechanical revolution) and the current A/D converters (14-bit resolution over a 50 A measurement span) were also included. All the results presented in this section are relative to speed and current error bounds equal to $e_{\omega, \max } / \omega_{N} \approx 0.018$ and $e_{d, \max } / I_{N} \approx 0.02$, respectively.

\section{A. Dynamic response}

The performances of the proposed HDPC under unloaded transient conditions are shown in Fig. 5a. The reported results refer to a speed ramp-up test from zero up to $\approx 15 \%$ of the nominal speed. Both the speed and $d$-axis current are regulated within their error bands during the whole transient. The $q$-axis current is different from zero only during the speed ramp, since no load torque is applied to the motor. The current ripple is satisfactory, being limited to few percent of the nominal current. As expected, the scaling factor $\kappa$ is adjusted accordingly to the motor speed, to compensate for the variation of the back electromotive force.

The robustness of the proposed HPDC against a sudden load torque variation is illustrated in Fig. 5b. The test is performed by applying a load torque equal to one-third of the nominal torque. The HDPC reacts with a fast dynamics, typical of a predictive control, with only a small overshot but a relatively large drop when the load torque is removed. This is due to the fact that the speed signal is always close to the upper bound of the error band. Hence, when a load torque is applied, the speed decrease, but the control does not react because the speed signal remains within the error band. On the other hand, when the load torque is removed, the speed tends at first to increase (small overshoot). This implies that the speed exceeds the upper limit of the band almost immediately. The control then reacts very quickly (as typical of finite-state control) by bringing again the speed signal within the error band. Last,
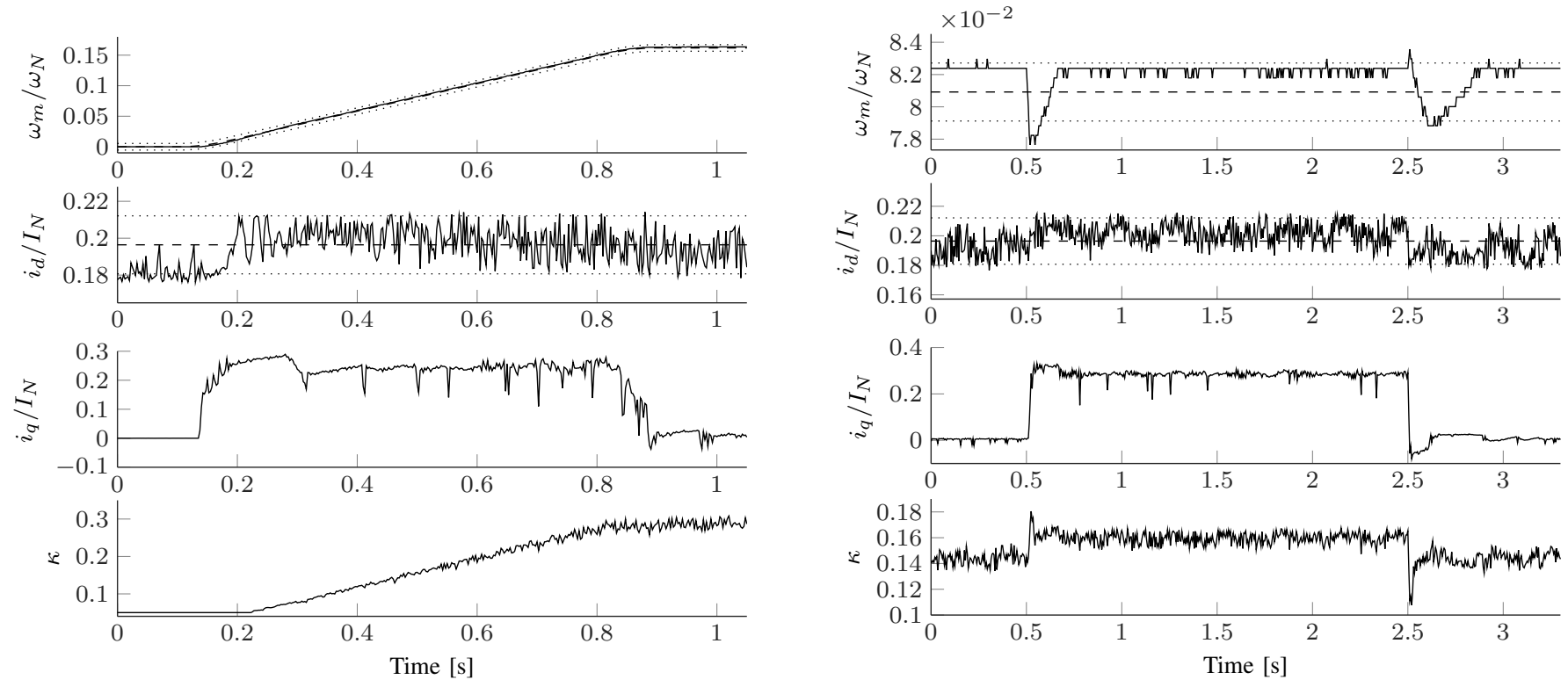

(a)

(b)

Fig. 5. Simulation results: dynamic response in a ramp-up test (left) and load torque rejection test (right). 
note that the factor $\kappa$ is adjusted according to the increased current demand.

\section{B. Current ripple reduction}

The benefits introduced by the scaled-state mechanism in terms of current ripple mitigation are illustrated in Fig. 6. The results were obtained by measuring the $q$-axis current at steady state conditions, with a speed reference $\omega_{m}^{*} / \omega_{N}=0.08$ and a constant load torque equal to one-third of the nominal torque (similar conditions to those used for the test of Fig. 5b). When the scaled-state mechanism is enabled (Fig. 6b), a one-order magnitude reduction of the current ripple amplitude (standard deviation - std dev) is noticed, compared to a situation where a full DC link voltage is used (Fig. 6a). The reduction of the current ripple can be further appreciated in Fig. 7, which shows the power spectral densities of the $q$-axis current evaluated in the two cases. Further to the current ripple reduction, the plot allows to predict some benefits in terms of acoustic noise emission, too. In fact, a limit cycle in the audible range $(\approx 400 \mathrm{~Hz})$ can be noticed in the current spectrum when full voltage state vectors are used. The limit cycle disappears when the scaled-state mechanism is enabled. The residual current "spikes" that can be noticed in Fig. 6b even after the introduction of the scaled state mechanism are deliberately generated by the HDPC, as evident from the expanded view around a single "spike" shown in Fig. 8. In practice, whenever the speed measurement exceeds the error band, the HDPC triggers a large current variation that quickly brings the motor speed back within the error band. This behaviour is the result of prioritising the current/torque dynamics in the hierarchical decisional process conceived in Sec. III-D. A different strategy, e.g. that prioritises the reduction of switching losses [12] [13], could force the application of a voltage vector adjacent to that applied at the previous step. This would smooth the current

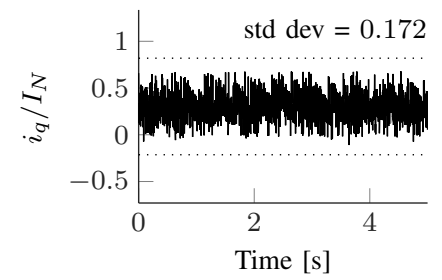

(a) without $\kappa$ adjustment

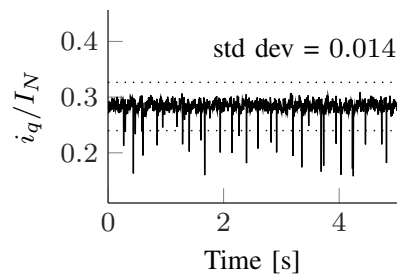

(b) with $\kappa$ adjustment
Fig. 6. Simulation results: reduction of $q$-axis current ripple. Dotted horizontal lines delimit the $\pm 3 \sigma$ region.

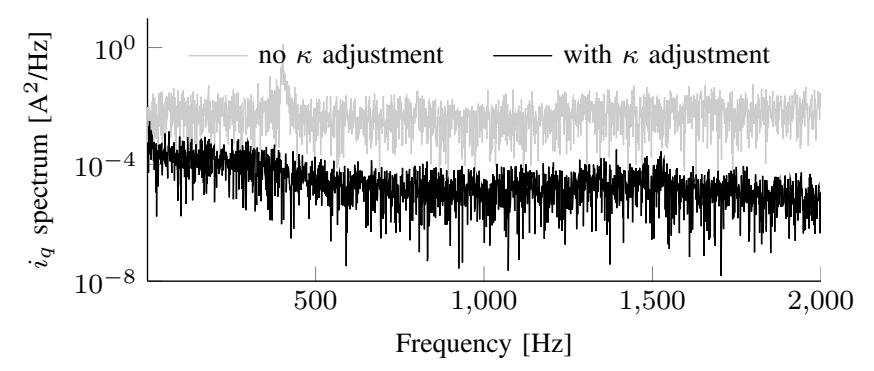

Fig. 7. Effects of scaling in the $i_{q}$ spectrum.
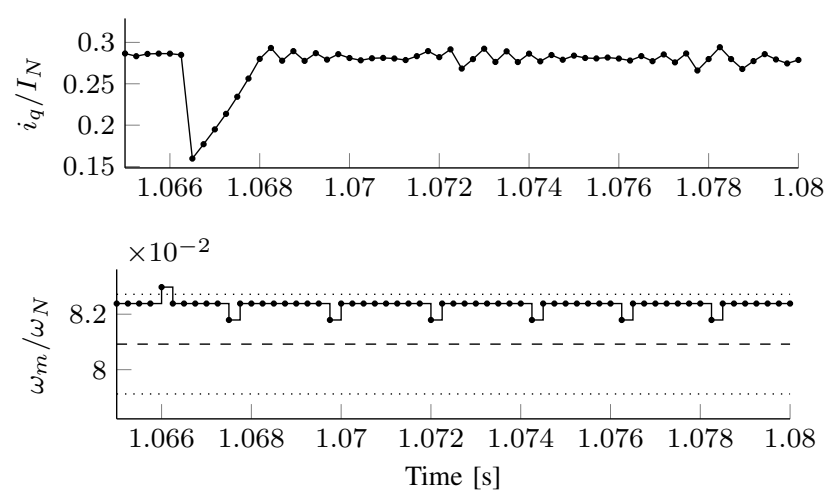

Fig. 8. Correlation between the speed error and the current spikes of Fig. $6 \mathrm{~b}$. The speed error band is delimited by horizontal dotted lines.

transients, at the expense of a slower speed control dynamics.

\section{Model accuracy}

Regarding the sensitivity of the proposed control to parameters uncertainty, it is known that in the MPC scenario, the HDPC is somewhat rough, since it selects the voltage control input among (few) state vectors whose amplitude is much greater than that strictly necessary. The proposed scaling policy mitigates this roughness, while preserving the principle. Therefore, a certain degree of robustness is maintained. Intuitively, if the variable used to select the best voltage vector input (1 out of 14 ) is slightly wrong due to parameters mismatch, the effects are evident only if the error is high enough to force a shift in the decision - while it is otherwise irrelevant. The effects of a multiplicative uncertainty were investigated by modifying the flux to current maps used in the SynRM model, according to the following equations

$$
i_{d}=\left(1+\delta_{d}\right) f_{d}\left(\lambda_{d}, \lambda_{q}\right), \quad i_{q}=\left(1+\delta_{q}\right) f_{q}\left(\lambda_{d}, \lambda_{q}\right)
$$

where $\delta_{d}$ and $\delta_{q}$ represent the relative uncertainties with respect to the nominal current-flux tables. A $\pm 20 \%$ uncertainty was supposed, and the uncertainties were applied individually, i.e. one at a time. Some simulation results are reported in Fig. 9 for a $\delta_{d}=+20 \%$ uncertainty. Very similar results are obtained for $\delta_{d}=-20 \%$ and for $\delta_{q}=+20 \%$. In all instances, the control response was satisfactory, with both the speed and $d$-axis current regulated within their error bands. Of course, as in any MPC system, excessive uncertainty is surely detrimental, and the control action abruptly deteriorates.

It is also worth to analyse whether the model approximations made in the model discretization of Sec. II-B are acceptable or not. Figure 10 reports the speed and currents prediction errors, respectively $\tilde{\omega}_{m}=\omega_{m, k+2}-\hat{\omega}_{m, k+2}$ and prediction errors $\tilde{i}_{d q}=i_{d q, k+2}-\hat{i}_{d q, k+2}$, obtained during the load rejection test. Both simulations and experimental results are reported. The prediction errors are very small (less than $1 \%$ of the nominal values), so that it can be reasonably concluded that the approximations introduced in Sec. II do not affect the prediction accuracy. 

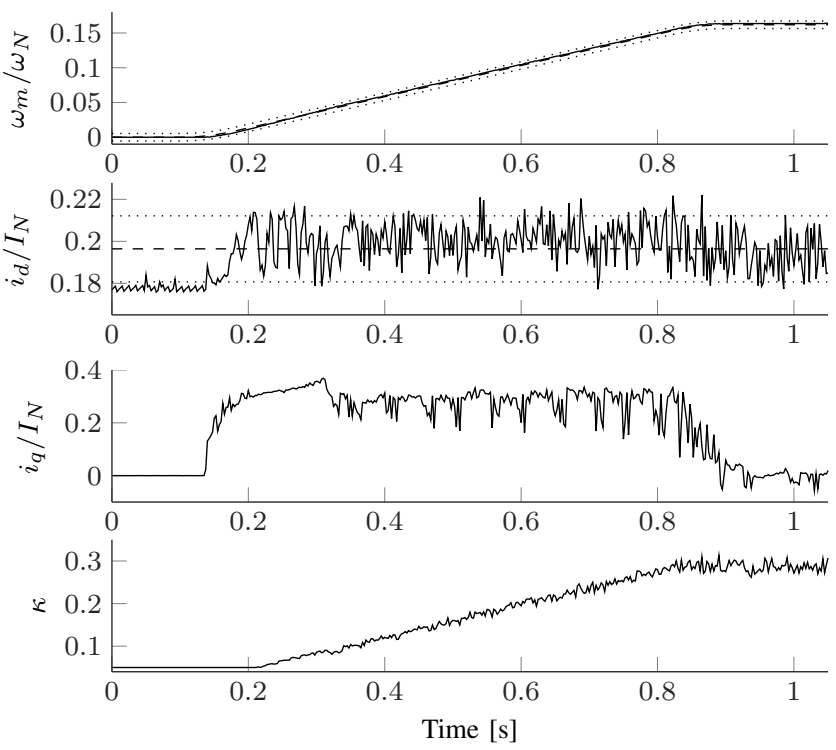

(a)
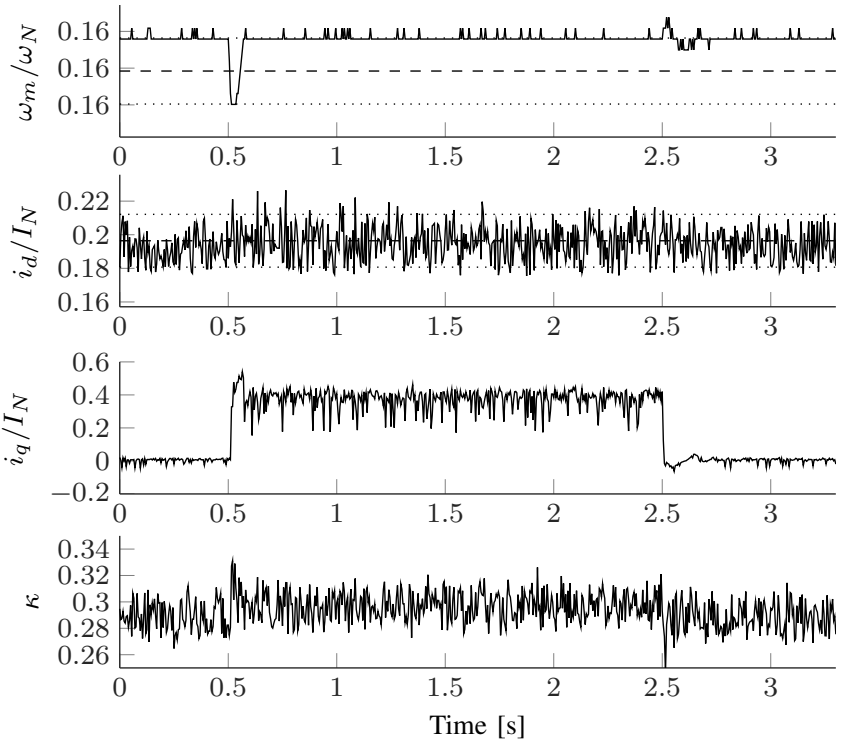

(b)

Fig. 9. Simulation results: robustness against variations of the magnetic flux $\left(\delta_{d}=+20 \%\right)$.
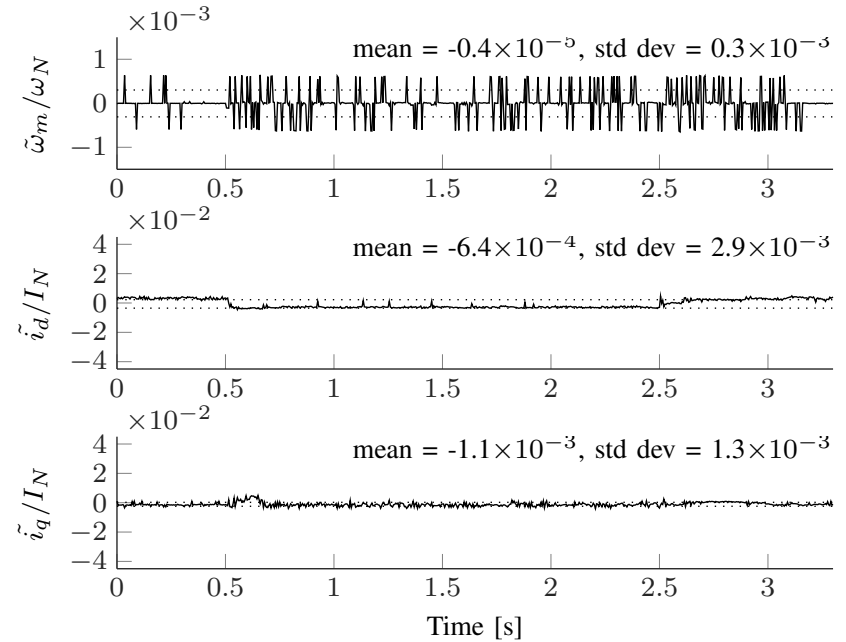

(a)
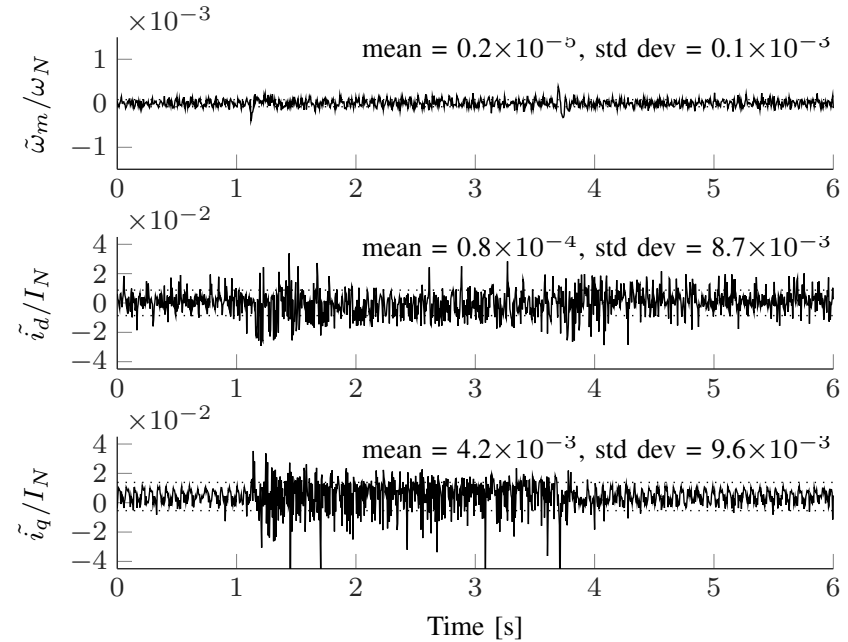

(b)

Fig. 10. Estimation errors in the load torque rejection test: simulation (left) vs experimental (right) results. Dotted horizontal lines delimit the $\pm 3 \sigma$ region.

\section{EXPERIMENTAL RESULTS}

\section{A. Experimental setup}

The test bench used for the experiments was composed by a back-to-back connection of an 11-kW SynRM prototype and an 11-kW Interior Permanent Magnet (IPM) motor acting as a dynamometer. A picture of the test bench is reported in Fig. 11 (IPM on the left side, SynRM on the right). A torque meter (under the black protection cover in Fig. 11) is connected between the two machines. The IPM torque is regulated via an off-the-shelf 22-kW ABB ACS850 (upper right side of the picture). The SynRM prototype is connected to the power unit of an ABB ACS850, where the control board has been replaced by a custom interface and connected to an OPAL-RT Technologies OP5600 system (black box on the left upper side of the figure). The OP5600 is equipped with a quad-core Intel
DSP processor at $2.4 \mathrm{GHz}$ and a Virtex 6 FPGA. The phase currents and the DC-bus voltage are measured with a custom measurement box (the grey box with a fan behind the load machine) and connected to the A/D converters of the OP5600. The digital I/O of the OP5600 are used to communicate with the ACS850 power unit through the custom interface.

The phase voltages used in the algorithms were obtained through estimation by an accurate compensation of the inverter non-linearities [23]. The reference voltages were used instead of the missing voltage measurements.

\section{B. HDPC implementation details}

The OP5600 system offers either a DSP or an FPGA implementation possibility for the HDPC algorithm. In this case, the FPGA was used only for low-level control procedures 


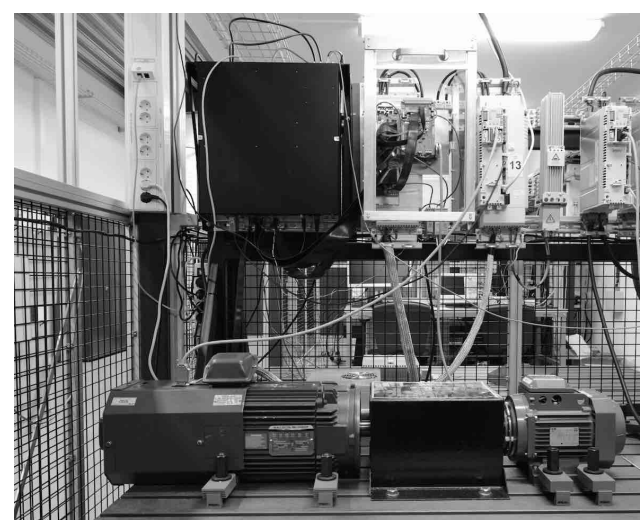

Fig. 11. Overview of the experimental setup.

(voltage modulation, I/O handling, protections), while the prediction and selection of the voltages was performed at DSP level. With this solution, the overall time required to perform the HDPC algorithm on the DSP was kept within $10.5 \%$ of the complete control cycle of $250 \mu \mathrm{s}$, leaving considerable resources available for other control purposes.

\section{Dynamic response}

The HDPC response during a ramp-up test, under the same conditions of Fig. 5a, is shown in Fig. 12a. Differently from simulations, an extra low-pass filtering of the scaling factor $\kappa$ was required to improve the rejection to measurement noise in the computation of (27), (28). Without any filtering action, the chattering on $\kappa$ would render the entire algorithm extremely noisy and prone to instabilities. This explains the smoother response of the factor $\kappa$ in Fig. 12, compared to that of Fig. 5.

It is also worth to note that during transient the $i_{d}$ current regulation error is within the specified bound, while there is a
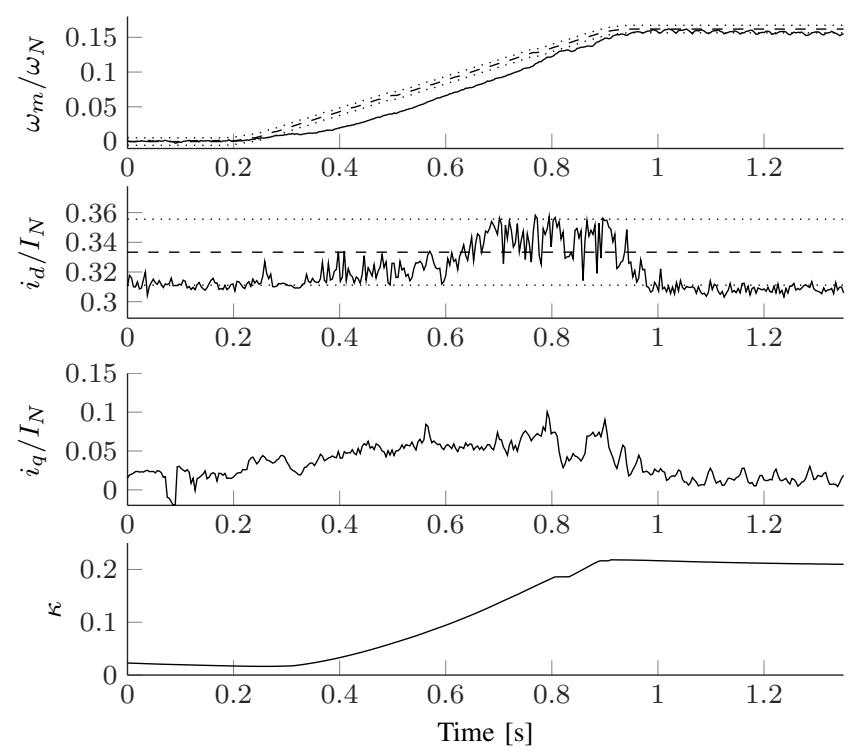

(a) speed error greater than expected during the ramping up. The sluggishness of the experimental speed response (compared to the simulated response shown in Fig. 5a) can be ascribed to the slower dynamics of $\kappa$, which is a little under-estimated with respect to the real need. On the other hand, this results in a smoother behaviour of the $i_{q}$ current during the same transient. This is a typical engineering trade-off that can be settled during the fine tuning of the drive.

\section{Robustness to load variations}

The results of a rejection test to a sudden load torque variation are reported in Fig. 12b. The test is performed at steady state speed, by applying a constant load torque equal to one-third of the nominal torque. Except for a very short transient, the speed is maintained below the prefixed bound during the whole load torque step. The $d$-axis current ripple is also below the bound, and the $q$-axis current ripple, whose minimisation has less priority in the hierarchical decisional structure, is below $25 \%$ of the nominal current.

\section{CONCLUSIONS}

A novel direct predictive control scheme for synchronous reluctance motors was presented in the paper. Its main advantages compared to a conventional scheme can be summarised as follows: (1) the introduction of an easier and more straightforward way to set priorities among the constraints of the predictive control formulation, for the sake of an experience-guided tuning, thanks to the adoption of a hierarchical decisional process for the selection of the optimal state voltage vector; (2) the capability of generating less current ripple without increasing the number of inverter states, as would be necessary in case of a multi-level inverter, thanks to the introduction of the concept of "scaled-states".
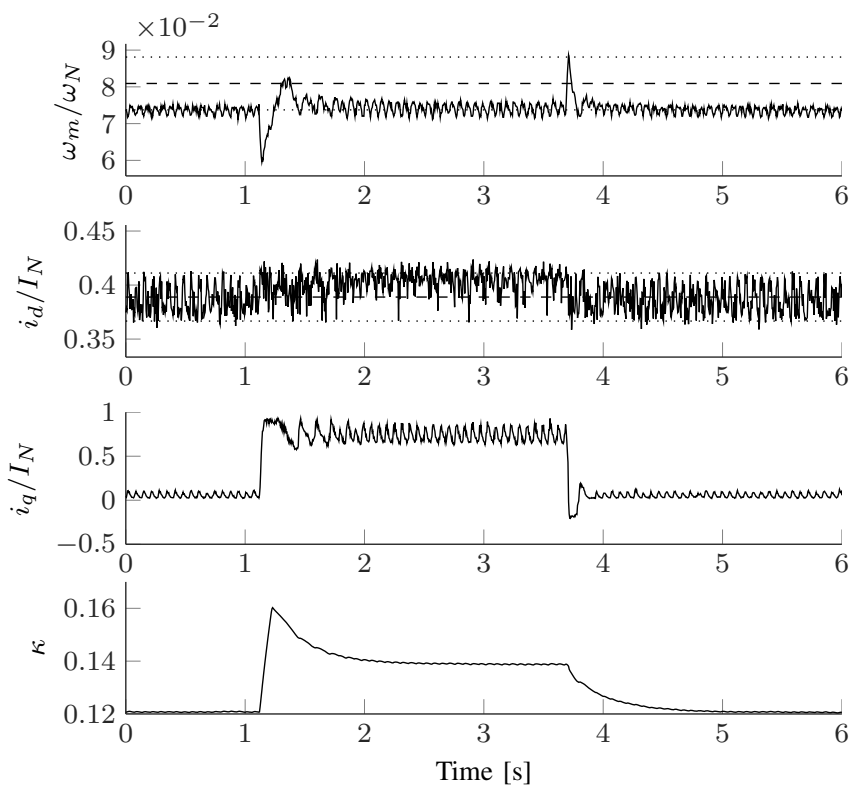

(b)

Fig. 12. Experimental results: ramp-up test (left); load torque disturbance rejection test (right). 
It is important to highlight that in all the experimental test reported in the paper, the drive was operated at the nominal DC bus voltage of $560 \mathrm{~V}$ and at a switching frequency of $4 \mathrm{kHz}$, that are particularly heavy conditions for predictive control, often underestimated in many technical papers on the subject. The effectiveness of the proposed design was proved by both simulation and experimental tests performed on an industrial $11 \mathrm{~kW}$ SynRM prototype. The results confirm the good control performances with limited current ripple, low switching losses and simple algorithm implementation. All these factors are particularly appreciated in medium to high power drives applications, where an high switching frequency poses serious issues of hardware circuitry and efficiency.

\section{APPENDIX}

The main parameters of the SynRM prototype are reported in Tab. I. The inertia of the whole test bench (SynRM + IPM + torque meter) was of $0.032 \mathrm{kgm}^{2}$.

TABLE I

11-kW SYNRM PARAMETERS.

\begin{tabular}{ccc}
\hline Stator resistance & $R$ & $0.72 \Omega$ \\
Pole pairs & $p$ & 2 \\
Nominal voltage & $U_{N}$ & $400 \mathrm{~V}$ \\
Nominal current & $I_{N}$ & $18 \mathrm{~A}$ \\
Nominal speed & $\omega_{N}$ & $650 \mathrm{rad} / \mathrm{s}$ \\
Nominal torque & $\tau_{N}$ & $17 \mathrm{Nm}$ \\
Mechanical inertia & $J_{m, N}$ & $0.0035 \mathrm{kgm}^{2}$ \\
\hline
\end{tabular}

\section{REFERENCES}

[1] F. Morel, X. Lin-Shi, J.-M. Retif, B. Allard, and C. Buttay, "A comparative study of predictive current control schemes for a permanent-magnet synchronous machine drive," IEEE Trans. Ind. Electron., vol. 56, no. 7, pp. 2715-2728, July 2009.

[2] M. Zigliotto, "Permanent magnet synchronous motor drives," in Power Electronic Converters and Systems - Frontiers and applications, 1st ed., A.M.Trzynadlowski, Ed. London, UK: IET, Power and Energy Series, 2015, pp. 313-332.

[3] J. Rodriguez and P. Cortes, Predictive Control of Power Converters and Electrical Drives. Wiley-IEEE Press, 2012.

[4] K. Belda and D. Vosmik, "Explicit generalized predictive control of speed and position of PMSM drives," IEEE Trans. Ind. Electron., vol. PP, no. $99,2016$.

[5] E. Camacho and C. Alba, Model Predictive Control, ser. Advanced Textbooks in Control and Signal Processing. Springer London, 2013.

[6] E. Fuentes, C. Silva, and J. Yuz, "Predictive Speed Control of a TwoMass System Driven by a Permanent Magnet Synchronous Motor," IEEE Trans. Ind. Electron., vol. 59, no. 7, pp. 2840-2848, July 2012.

[7] J. M. Maciejowski, Predictive control with constraints. Essex, England: Prentice Hall, 2002.

[8] S. Bolognani, S. Bolognani, L. Peretti, and M. Zigliotto, "Design and implementation of model predictive control for electrical motor drives," IEEE Trans. Ind. Electron., vol. 56, no. 6, pp. 1925-1936, June 2009.

[9] M. Cychowski, K. Szabat, and T. Orlowska-Kowalska, "Constrained model predictive control of the drive system with mechanical elasticity," IEEE Trans. Ind. Electron., vol. 56, no. 6, pp. 1963-1973, June 2009.

[10] J. Rodriguez, M. Kazmierkowski, J. Espinoza, P. Zanchetta, H. Abu-Rub, H. Young, and C. Rojas, "State of the Art of Finite Control Set Model Predictive Control in Power Electronics," IEEE Trans. Ind. Informat., vol. 9, no. 2, pp. 1003-1016, May 2013.

[11] P. Karamanakos, T. Geyer, N. Oikonomou, F. Kieferndorf, and S. Manias, "Direct model predictive control: A review of strategies that achieve long prediction intervals for power electronics," IEEE Ind. Electron. Mag., vol. 8, no. 1, pp. 32-43, March 2014.
[12] M. Preindl and S. Bolognani, "Model Predictive Direct Torque Control With Finite Control Set for PMSM Drive Systems, Part 1: Maximum Torque Per Ampere Operation," IEEE Trans. Ind. Informat., vol. 9, no. 4, pp. 1912-1921, Nov 2013.

[13] M. Preindl and S. Bolognani, "Model Predictive Direct Torque Control With Finite Control Set for PMSM Drive Systems, Part 2: Field Weakening Operation," IEEE Trans. Ind. Informat., vol. 9, no. 2, pp. 648-657, May 2013.

[14] C.-S. Lim, E. Levi, M. Jones, N. Rahim, and W.-P. Hew, "A comparative study of synchronous current control schemes based on FCS-MPC and PI-PWM for a two-motor three-phase drive," IEEE Trans. Ind. Electron. vol. 61, no. 8, pp. 3867-3878, Aug 2014.

[15] M. Siami, A. Abbaszadeh, D. Arab Khaburi, and J. Rodriguez, "Robustness improvement of predictive current control using prediction error correction for permanent magnet synchronous machines," IEEE Trans. Ind. Electron., vol. PP, no. 99, 2016.

[16] X. Lin-Shi, F. Morel, A. Llor, B. Allard, and J.-M. Retif, "Implementation of hybrid control for motor drives," IEEE Trans. Ind. Electron., vol. 54, no. 4, pp. 1946-1952, Aug 2007.

[17] E. Levi, "Advances in converter control and innovative exploitation of additional degrees of freedom for multiphase machines," IEEE Trans. Ind. Electron., vol. PP, no. 99, pp. 1-1, 2015.

[18] S. Vazquez, J. Leon, L. Franquelo, J. Carrasco, O. Martinez, J. Rodriguez, P. Cortes, and S. Kouro, "Model predictive control with constant switching frequency using a discrete space vector modulation with virtual state vectors," in ICIT 2009, Feb 10-13 2009, pp. 1-6.

[19] E. Schrijver and J. van Dijk, "Disturbance Observers for Rigid Mechanical Systems: Equivalence, Stability, and Design,” J. of Dyn. Sys., Meas., and Cont., vol. 124, no. 4, pp. 539-548, 122002.

[20] L. Peretti and D. Svechkarenko, "Self-commissioning procedure for inductance estimation in an electrical machine," Feb. 6 2013, eP Patent App. EP20,110,176,085.

[21] E. Armando, R. Bojoi, P. Guglielmi, G. Pellegrino, and M. Pastorelli, "Experimental identification of the magnetic model of synchronous machines," IEEE Trans. Ind. Appl., vol. 49, no. 5, pp. 2116-2125, Sept 2013.

[22] G. Pellegrino, B. Boazzo, and T. Jahns, "Magnetic model selfidentification for pm synchronous machine drives," IEEE Trans. Ind. Appl., vol. 51, no. 3, pp. 2246-2254, May 2015.

[23] M. Carraro and M. Zigliotto, "Automatic parameter identification of inverter-fed induction motors at standstill," IEEE Trans. Ind. Electron., vol. 61, no. 9, pp. 4605-4613, Sept 2014.

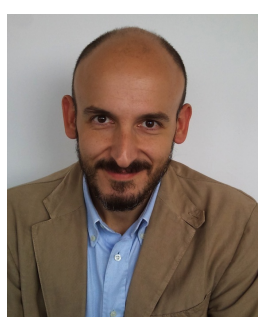

Riccardo Antonello (M '02) received the Laurea degree (cum laude) in Computer Engineering and the Ph.D degree in Automatic Control from the University of Padova, Italy, in 2002 and 2006 respectively. From 2006 to 2010 he has been a research associate at the Dept. of Mechanical and Structural Engineering, University of Trento, Italy. From 2010, he joined the Dept. of Management and Engineering, University of Padova. His current research interests lie in the areas of control systems, electric drives and mechatronics.

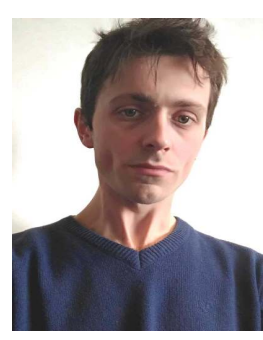

Matteo Carraro received the M.Sc. degree in Automation Engineering and the Ph.D. degree in Mechatronics Engineering from the University of Padova, Padova, Italy, in 2010 and 2014 respectively. In 2013 he was a Visiting Ph.D. Student at ABB Corporate Research Center, Department of Power Technologies, Västerås, Sweden. He is currently with Sael Srl, Torri di Quartesolo (VI), IT. His research interests are in the areas of sensorless control algorithms and parameter estimation strategies for AC motor drives. 


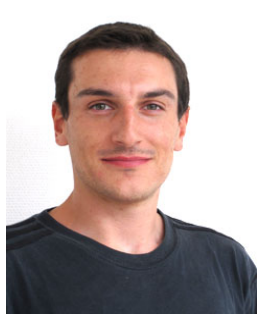

Luca Peretti received the M.Sc. in electronic engineering from the University of Udine, Italy, in 2005, and the Ph.D. in Mechatronics and Industria Systems from the University of Padova, Italy, in 2009. From January 2009 to July 2010 he held a Post Doctoral position at the University of Padova. In August 2010, he joined ABB Corporate Research in Västerås, Sweden, where he is currently working as a principal scientist. His activities target the field of electrical machines and power electronics for drives, with particular focus on machine parameter estimation, sensorless control and algorithms for diagnostics in the industrial and wind energy scenarios. Luca Peretti is a member of the IET.

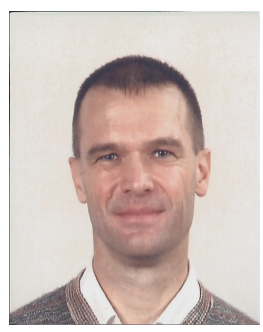

Mauro Zigliotto (M'98) is a native of Vicenza, Italy. He is full professor of Electrical Machines and Drives at University of Padova, Italy and head of the Electric Drives Laboratory in Vicenza, Italy. Advanced control strategies and self-commisioning for ac motors are Prof. Zigliotto's main research interests. He is the secretary of the IEEE IAS-IESPELS North Italy Joint Chapter. 